\title{
Breast implant-associated anaplastic large cell lymphoma (BIA-ALCL) in Poland: analysis of patient series and practical guidelines for breast surgeons
}

\author{
Piotr Pluta ${ }^{1}$, Agnieszka Giza ${ }^{2}$, Małgorzata Kolenda ${ }^{3}$, Wojciech Fendler, ${ }^{4,5}$, Marcin Braun ${ }^{6}$, \\ Cezary Chudobiński , Justyna Chałubińska-Fendler ${ }^{6}$, Michał Araszkiewicz ${ }^{8}$, Karolina Loga ${ }^{9}$, \\ Lubomir Lembas ${ }^{10}$, Magdalena Witkowska ${ }^{11}$, Agnieszka Pluta ${ }^{12}$, Jerzy Kolasiński ${ }^{3}$, Paweł Basta ${ }^{13}$,

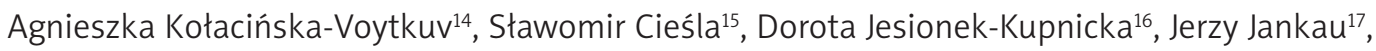 \\ Maciej Kuczyński ${ }^{18}$, Ewa Kalinka ${ }^{19}$, Marek Zadrożny ${ }^{1}$, Dawid Murawa ${ }^{15}$
}

\author{
1Department of Surgical Oncology and Breast Diseases, Polish Mother's Memorial \\ Hospital - Research Institute, Lodz, Poland \\ ${ }^{2}$ Department of Hematology, Jagiellonian University Medical College, Krakow, Poland \\ ${ }^{3}$ Klinika Kolasinski, Swarzedz, Poland \\ ${ }^{4}$ Department of Radiation Oncology, Harvard Medical School, Dana-Farber Cancer \\ Institute, Boston, Massachusetts, USA \\ ${ }_{5}^{5}$ Department of Biostatistics and Translational Medicine, Medical University of Lodz, \\ Lodz, Poland \\ ${ }^{6}$ Department of Radiotherapy, Military Institute of Medicine, Warsaw, Poland \\ ${ }^{7}$ Department of Radiology, Regional Oncology Centre, Copernicus Memorial Hospital, \\ Lodz, Poland \\ ${ }^{8}$ Legal Advisor's Office, Polish Mother's Memorial Hospital - Research Institute, Lodz, \\ Poland \\ ${ }^{9}$ Department of Radiotherapy and General Oncology, Regional Oncology Centre, \\ Copernicus Memorial Hospital in Lodz, Medical University of Lodz, Lodz, Poland \\ ${ }^{10}$ Private Practice Lubomir Lembas, Warsaw, Poland \\ ${ }^{11}$ Department of Experimental Hematology, Medical University of Lodz, Lodz, Poland \\ ${ }^{12}$ Department of Hematology, Medical University of Lodz, Lodz, Poland \\ ${ }^{13}$ Departament of Gynecology and Oncology, Jagiellonian University, Medical College \\ Breast Unit of University Hospital, Krakow, Poland \\ ${ }^{14}$ Department of Head and Neck Cancer Surgery, Breast Cancer Unit, Medical \\ University of Lodz, Lodz, Poland \\ ${ }^{15} \mathrm{Clinic}$ of General Surgery and Surgical Oncology, Faculty of Medicine and Health \\ Sciences University, Zielona Gora, Poland \\ ${ }^{16}$ Department of Pathology, Chair of Oncology, Medical University of Lodz, Lodz, \\ Poland \\ ${ }^{17}$ Department of Plastic Surgery, Gdansk Medical University, Gansk, Poland \\ ${ }^{18}$ Dr Maciej Kuczyński Plastic Surgery Center, Lublin, Poland \\ ${ }^{19}$ Department of Oncology, Polish Mother's Memorial Hospital - Research Institute, \\ Lodz, Poland
}

Submitted: 12 July 2020

Accepted: 7 September 2020

Arch Med Sci

DOI: https://doi.org/10.5114/aoms.2020.100637

Copyright (c) 2020 Termedia \& Banach

\begin{abstract}
Introduction: Although breast implant-associated anaplastic large cell lymphoma (BIA-ALCL) is infrequent, with less than 1000 noted cases worldwide, patients consenting for breast implant surgery should be aware of its risk. We describe the first Polish multicenter case-series data on BIA-ALCL patients and present diagnostic and treatment recommendation for breast surgeons. Material and methods: In cooperation with the Polish Society of Surgical Oncology and Polish Lymphoma Research Group, we collected BIA-ALCL cases in Poland.
\end{abstract}

\author{
Corresponding author: \\ Piotr Pluta \\ Department of Surgical \\ Oncology and \\ Breast Diseases \\ Polish Mother's \\ Memorial Hospital - \\ Research Institute \\ Lodz, Poland \\ Phone: +48604 188815 \\ E-mail: \\ drpiotrpluta@gmail.com
}


Results: We retrospectively reviewed clinical data of seven BIA-ALCL patients, diagnosed between July 2013 and November 2019. The median time from implant placement to the first BIA-ALCL symptoms was 65 months (range: 33-96 months). All the patients were exposed to textured implants at presentation. Capsulectomy with implant removal was performed in all the patients with immediate reimplantation in 2 cases. In a median follow-up of 19 months (range 5-81 months), there was no recurrence and all the patients stayed alive. Between 2013 and 2019, the incidence of BIA-ALCL in Polish female population age 30 and above ranged from 0 to 0.021/100 000/year. Conclusions: BIA-ALCL is scarce in the Polish population. In a short-term follow-up, patients' prognosis remains excellent. Due to the withdrawal of roughly textured implants from the market and the exclusion of likely the most potent etiologic factor, it might be expected that the incidence of BIA$\mathrm{ALCL}$ will become even rarer.

Key words: guidelines, incidence, breast implants, breast lymphoma, breast implant-associated anaplastic large cell lymphoma.

\section{Introduction}

Silicone implants and expanders are commonly used in aesthetic and reconstructive breast surgery. Every year, plastic surgeons perform over 1.8 million breast augmentations globally, which makes this procedure the most common in the whole surgical esthetic panel [1]. Implant-based reconstructions have become a leading technique in both risk-reducing mastectomies and postcurative mastectomies, surpassing in number autologous breast reconstructions [2, 3]. The wide acceptance of silicon prostheses is based on the data supporting their health harmlessness. In the past, main concerns considered the risk of breast cancer in implant-exposed patients. It resulted from the data on the carcinogenetic role of polyurethane breakdown products in animal models, which undermined the safety of silicone polyurethane (PU) coated implants [4, 5]. These effects have never been proved in humans and until recently, breast implants were considered as causally unrelated to malignancies.

In 1997 Keech and Creech presented a case of a female patient after breast augmentation with an anaplastic large cell lymphoma (ALCL) mass in proximity to the saline-filled implant [6]. Since then, growing numbers of patients have proved the link between breast implants and ALCL in surrounding tissues. In 2016, the WHO classification separated breast implant-associated anaplastic large cell lymphoma (BIA-ALCL) as a novel disease entity [7]. This malignancy, by definition, belongs to the non-Hodgkin lymphomas and results from the clonal proliferation of T-cells in peri-implant fluid or the fibrous capsule. Histologically BIAALCL represents large pleomorphic cells, immunepositive for CD30 T-cell antigens and negative for ALK proteins [8]. Prognosis in BIA-ALCL is favorable with 90\% 5-year overall survival; however, in approximately $15 \%$ of cases it extends beyond the breast, invading regional lymph nodes or occasionally forming distant metastases [9]. This outcome is similar to the skin ALCL but is in contrast to the systemic ALK-negative $\operatorname{ALCL}[10,11]$.

According to the American Society of Plastic Surgeons, 888 cases of BIA-ALCL were reported worldwide at the beginning of 2020, which makes BIA-ALCL a very uncommon disease compared to breast cancer and even to primary breast lymphomas $[12,13]$. The estimated prevalence of $\mathrm{ALCL}$ in the general population ranges from 1 to 9 per 100000 people [14]. The risk of ALCL located in the breast is much higher in women with implants compared to women with no implants or implant history. In 2018 de Boer et al. estimated the odds ratio of breast ALCL as 421.8 (95\% Cl: 52.6-3385.2; $p<0.001)$ for patients with breast implants [15]. Therefore, breast surgeons need information about the incidence of BIA-ALCL and practical guidance on how to diagnose and treat such patients. It is also patients' right to be informed of the risk of BIA-ALCL while consenting for breast implant surgery $[16,17]$. Likewise, patients exposed to rough surface implants expect surgeons' advice, as, since 2018, pharmaceutical companies have been recalling the macro-textured implants due to the increased risk of BIA-ALCL $[17,18]$.

The multidisciplinary character and rarity of this disease require a national task force for data collection and advice service. Herein, we describe the first Polish multicenter case-series data of BIAALCL patients and present diagnostic and treatment recommendation for breast surgeons.

\section{Material and methods}

To collect the data of all BIA-ALCL patients in Poland, we addressed our search request directly to the members of the Polish Society of Surgical Oncology, the Polish Society of Plastic, Reconstructive and Aesthetic Surgery and the Extranodal 
Section of Polish Lymphoma Research Group, collecting BIA-ALCL cases in Poland. Additionally, we discussed this project with Polish hematopathologists during the Lymphoma Forum of Excellence Pathology in Warsaw, on June $7^{\text {th }}$ to $8^{\text {th }}, 2019$.

The study includes BIA-ALCL cases reported until March 2020. The survey contained questions about clinical data and types of implants. Stage of the disease was determined according to the TNM staging system for solid cancer and the Ann Arbor system for non-Hodgkin lymphomas $[9,19]$. The follow-up data were obtained in March 2020 via phone calls.

The median time to BIA-ALCL occurrence was established from the implant insertion (the definitive implantation before diagnosis if multiple surgeries) to the first lymphoma symptoms. Median follow-up was assessed from the final surgical intervention for BIA-ALCL purposes to the last observation.

BIA-ALCL incidence was estimated in the female population age 30 and above, based on the data provided by Statistics Poland [20].

The Bioethics Committee of District Medical Council in Lodz has approved this study.

\section{Results}

We identified seven BIA-ALCL patients, diagnosed between July 2013 and November 2019. Median age at diagnosis was 46 (range: 30-64 years). Table I summarizes characteristics of the patients. The numbering of cases in the text refers to those in the table. Case 4 has previously been published [21].

All the patients were exposed to textured implant surgery for aesthetic or reconstructive purposes ( 5 and 2 patients, respectively). Breast reconstructions were performed using multiple types of implants. The manufacturers of implants associated with BIA-ALCL (the last implant before the symptoms in numerous exposures) were Allergan (5 cases including McGhan), and Mentor and Silimed in 1 patient each.

One patient with multiple implant exposure (case 4) had direct-to-implant immediate reconstruction after prophylactic mastectomy, resulting in a sequence of unsatisfactory outcomes, capsular contracture and ruptures. These complications required multiple revisions using Nagor, Mentor, McGhan and Allergan implants. Finally, bilateral capsulectomies and implants' replacement with Polytech were performed. The postoperative pathological assessment revealed BIA-ALCL in the unilateral breast, restricted to the capsule and peri-implant fluid. The patient refused any further treatment.

The second patient with multiple textured devices (case 6) underwent 2-stage breast reconstruction, in which both the expander and the implant had the same textured surface by Allergan.
She was the only patient with breast cancer history in our series.

Median time from implant placement to the first BIA-ALCL symptoms was 65 months (range: 33-96 months). In 4 out of 7 patients, unilateral breast swelling was the single symptom at presentation and in one it was accompanied by breast pain. Altogether, breast pain was reported by three patients. The remaining loco-regional symptoms included palpable mass in the breast or axilla and capsular contracture. One patient presented systemic symptoms such as weakness, fever, dyspnea and lymphadenopathy.

In pre-treatment imaging workup, breast ultrasound (US) confirmed peri-implant seroma in all the patients. Additionally, in 3 cases, breast magnetic resonance imaging (MRI) was performed; in three cases, the images were misinterpreted. In one case, MRI described suspicious capsule thickening which proved to be inflammation in final pathology. In 2 patients implants assumed to have ruptured were intact at the surgery. Increased 18-fluorodeoxyglucose (FDG) uptake in the breast or axillary lymph nodes was detected in 3 cases out of 4 preoperative positron emission tomography/computed tomography (PET/CT) imaging.

Ultrasound-guided fine-needle aspiration (FNA) biopsy was performed in 4 patients, and in all the cases, cytology, supplemented by immunocytochemical staining, revealed BIA-ALCL cells. The flow cytometry analysis confirmed the diagnosis in 1 case. The patient with systemic adenopathy underwent surgical biopsy of the neck lymph nodes, which established the diagnosis. In two cases, BIAALCL was revealed incidentally in the routine histopathology of postoperative specimens.

Primary surgery was performed in all the patients, excluding the patient with systemic symptoms and advanced disease, who had received preoperative chemotherapy followed by high-dose chemotherapy supported by autologous stem cell transplantation. Capsulectomy with implant removal was performed in all the cases; in 5 , it was a bilateral intervention, and in two a unilateral one. In two instances, immediate reimplantation was performed. Axillary node biopsy was complemented in two cases. One patient underwent re-excision of the breast wound due to the falsepositive postoperative PET/CT scans. Adjuvant chemotherapy was administered in one patient.

In a median follow-up of 19 months (range: 5-81 months), there was no recurrence, and all the patients stayed alive. Figure 1 demonstrates an exemplary clinical presentation.

Between 2013 and 2019, the annular incidence of BIA-ALCL among 100000 women age 30 and above ranged between 0 and 0.021 . Table II shows the detailed data. 


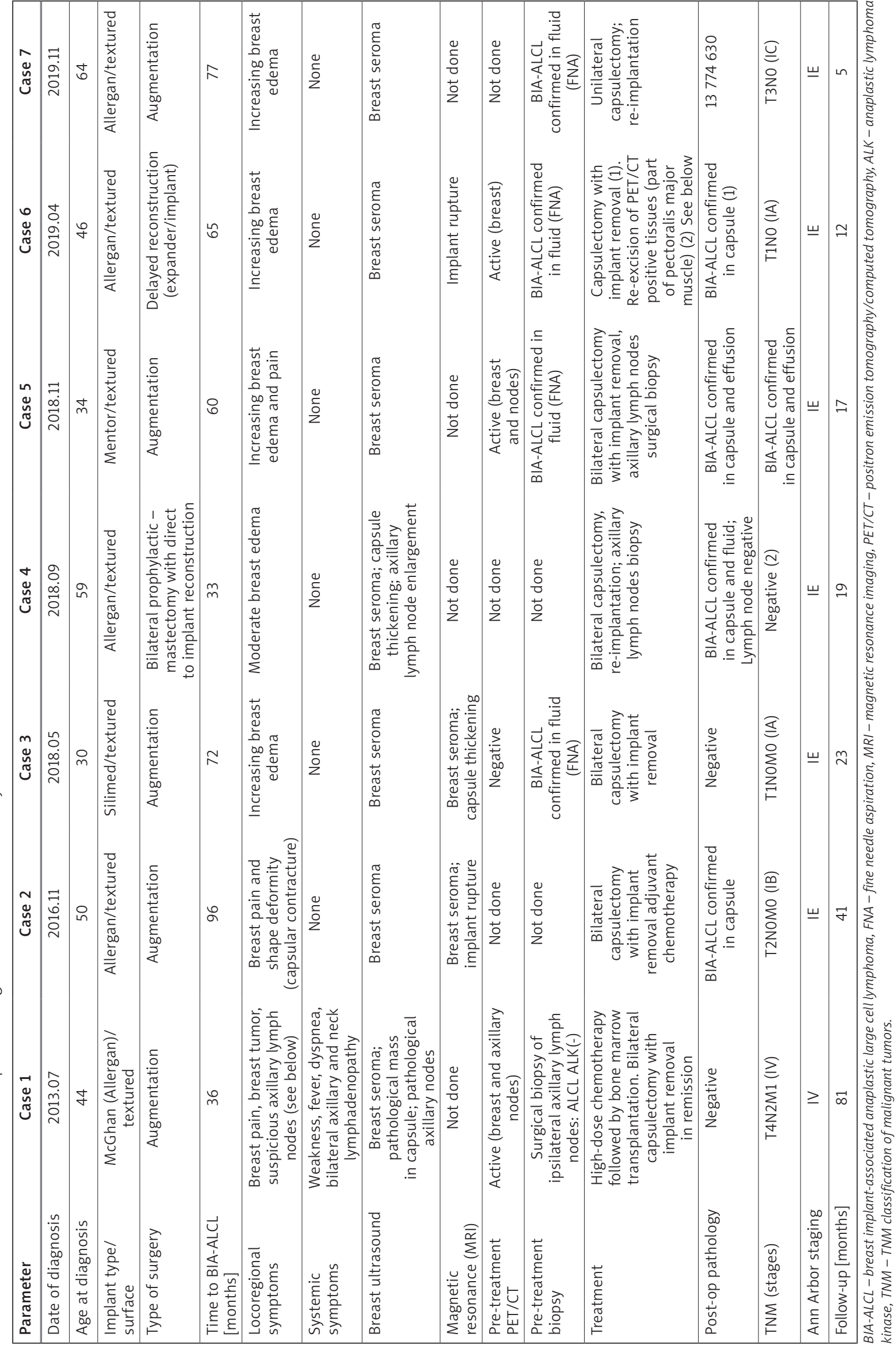



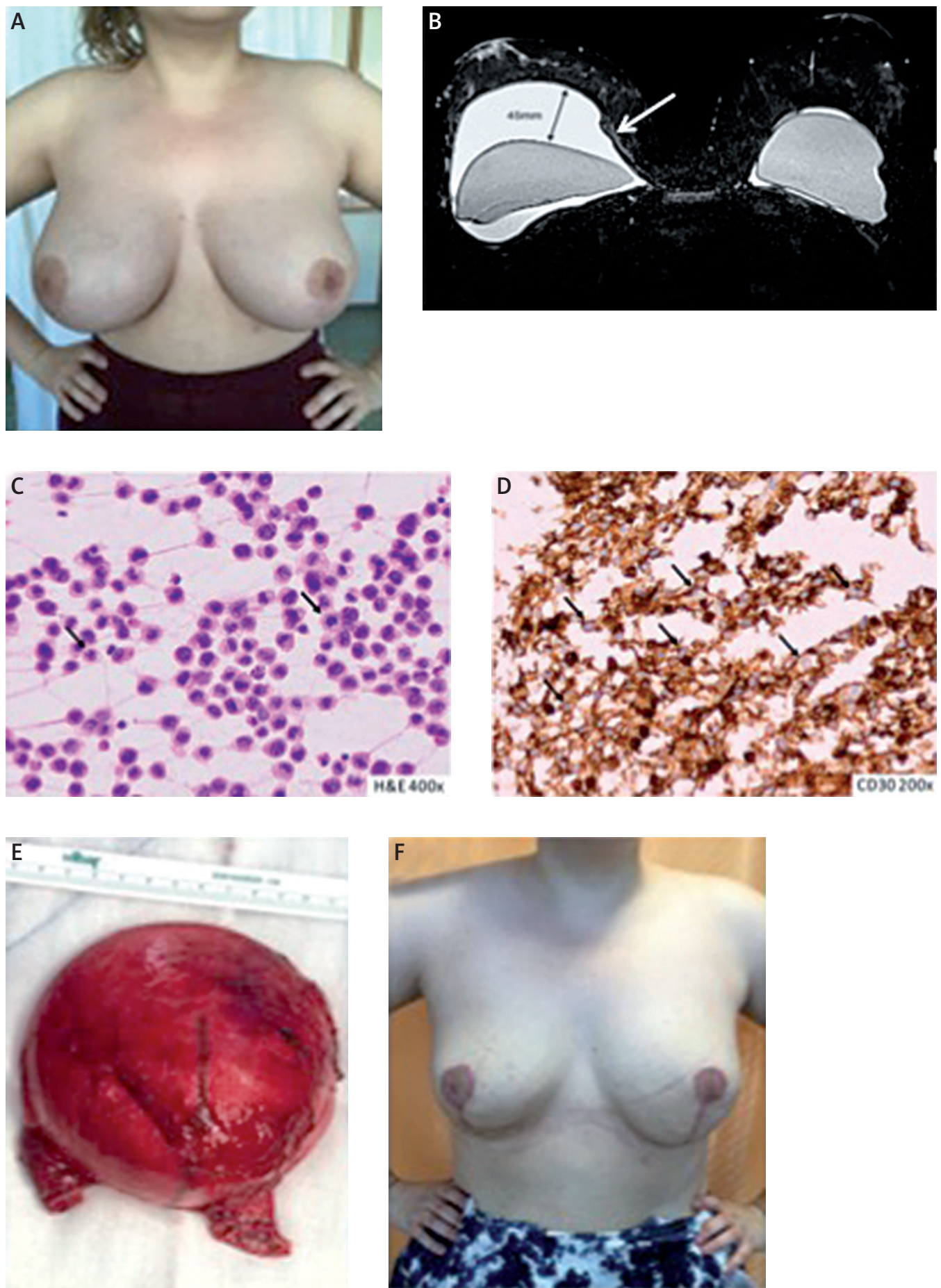

Figure 1. A patient with breast implant-associated anaplastic cell lymphoma (BIA-ALCL) (case 3). A thirty-year-old woman reported rapidly increasing swelling of the right breast (A). She negated other complaints. Six years earlier, she underwent bilateral augmentation with Silimed textured implants. Breast ultrasound revealed peri-implant seroma formation. Magnetic resonance imaging confirmed effusion layer $45 \mathrm{~mm}$ in width, covering the right implant; additionally, thickening of the fibrous capsule (white arrow) was noticed (B). Peri-implant layer on the left breast did not exceed $5 \mathrm{~mm}$. Hematoxylin and eosin staining of effusion smear disclosed the presence of large lymphoid cells, with irregular nuclei (black arrows) (C). Numeous atypical cells showed intense positive CD30 immunocytochemical staining (black arrows) (D). Preoperative positron emission tomography scans were negative. The patient underwent bilateral implants removal with capsulectomies and breast reductions. The peri-implant fibrous capsules were sent intact for pathological assessment $(\mathbf{E})$. The postoperative pathology did not disclose lymphoma invasions in either capsule, which settles the case to the stage IA according to the TNM classification and to IE in Ann Arbor staging for non-Hodgkin lymphomas [9, 17]. No adjuvant treatment was administrated. In 23 months of follow-up, the patient has remained recurrence-free; she has selected no other breast esthetics surgery (F) 
P. Pluta, A. Giza, M. Kolenda, W. Fendler, M. Braun, C. Chudobiński, J. Chałubińska-Fendler, M. Araszkiewicz, K. Loga, L. Lembas, M. Witkowska, A. Pluta, J. Kolasiński, P. Basta, A. Kołacińska-Voytkuv, S. Cieśla, D. Jesionek-Kupnicka, J. Jankau, M. Kuczyński, E. Kalinka, M. Zadrożny, D. Murawa

Table II. Annular incidence of BIA-ALCL in Polish female population age 30 and above between 2013 and 2019. Denominators retrieved from Statistics Poland [16]. Data for 2019 carried forward from 2018 as it is still unavailable

\begin{tabular}{|lccccccc|}
\hline \multicolumn{7}{|l}{ Variable } & \multicolumn{7}{c|}{ Year } & 2018 & 2019 \\
\cline { 2 - 9 } & 2013 & 2014 & 2015 & 2016 & 2017 & 2 \\
\hline $\begin{array}{l}\text { Number of patients } \\
\text { diagnosed with BIA-ALCL }\end{array}$ & 1 & 0 & 0 & 1 & 0 & 2 \\
\hline Number of women 30+ & 13242889 & 13381205 & 13499544 & 13609743 & 13696945 & 13774630 & 13774630 \\
\hline Incidence & 0.007 & 0 & 0 & 0.007 & 0 & 0.014 & 0.021 \\
\hline
\end{tabular}

BIA-ALCL - breast implant-associated anaplastic large cell lymphoma [18].

\section{Discussion}

Current studies indicate that the textured surface of the implant and bacterial contamination contribute to BIA-ALCL pathogenesis [22]. Compared to the smooth implants, the rough outer shell of the implants gives beneficial conditions for bacterial attachment and growth, which triggers the host chronic inflammatory response [23]. Consequently, the sustained T-cell activation may promote aberrant clones, leading to symptomatic BIA-ALCL [24].

The study of Loch-Wilkinson et al. confirmed that the higher surface area textured implants of Biocell (Allergan) and polyurethane (Silimed) markedly increase the risk of BIA-ALCL [25]. Similarly, in our study, six of seven patients were exposed to textured implants, which corresponds with the hypothesis of a role of such a surface in BIA-ALCL genesis. Presumably, the decision to recall the aggressive-textured implants from the world markets, including the EU, will cause this disease to become even more scarce $[18,26]$. Incorporating the aseptic procedure, e.g. a 14-point plan for implant placement, and further genetic research to select high-risk patients would fall within this optimistic scenario [27].

Clinical presentation of BIA-ALCL has usually developed within 7 to 10 years from implantation as unilateral breast swelling caused by spontaneous, non-inflammatory peri-prosthetic effusion [9, $22,23]$. This so-called "late and cold seroma" is not pathognomonic for BIA-ALCL, as it might result from subclinical infections or trauma [8]. Moreover, a thin liquid coating of the implant whose volume did not exceed $5-10 \mathrm{ml}$ is typical and does not require further tests [9]. In our series, onset of the disease was reported earlier, in a median time of implant exposure just under 5.5 years. Besides breast enlargement, breast pain was also often a reported symptom in our series. Palpable breast mass, the second frequent symptom of BIA-ALCL described in the literature, was observed only in a stage IV patient [8, 9, 22, 23].

There are several imaging techniques in detection of a peri-implant effusion or mass, including breast ultrasound (US), mammography, MRI, chest computed tomography (CT) and PET/CT [9, 22, 23, $28,29]$. In a retrospective study Adrada et al. assessed the effectiveness of these modalities in detection of effusion and mass in 44 BIA-ALCL patients [28]. Breast US and MRI achieve the highest sensitivity for effusion disclosure, exceeding $80 \%$. Except for PET/CT, no other technique gained over $50 \%$ sensitivity in mass detection. In our study, breast US revealed excessive peri-implant effusion in all the cases. Breast MRI was performed in 3 patients but their results were misleading regarding the capsule involvement and implant ruptures. It may show that breast MRI assessment in BIA-ALCL suspicion might be challenging, which makes it an addition to the routine US rather than an alternative. Still, a preoperative MRI serves as a tool to exclude breast cancer before the initiation of BIA-ALCL treatment.

None of our patients had preoperative mammography, which might be motivated by its limited role in augmented or reconstructed breasts [30]. Although mammography is a standard tool in breast cancer screening programs, it is insufficient to distinguish effusion from the mass [28, 31]. Similarly, CT chest scans were not made during preoperative workups in our series, as this modality does not belong to the routine breast imaging.

PET/CT imaging is the method of choice in the assessment of the disease staging, treatment response and follow-up in FDG avid lymphomas $[32,33]$. Notably, in BIA-ALCL, timing of PET/CT remains debatable, due to the increased risk of postbiopsy or post-surgery false-positive results $[9,33$, 34]. In our study, two of three preoperative PET/CT overestimated the nodes staging. Additionally, in one case, early postoperative PET/CT showed increased FDG uptake in the pectoralis major muscle, which led to the re-excision of the breast tissues. In this case, histopathological examination did not confirm residual lymphoma, which extends the pool of PET/CT false-positive results in our series. Therefore, we conclude that preoperative PET/CT imaging should be limited to patients with locally advanced or systemic symptoms of BIA-ALCL, who would benefit from primary chemotherapy. We 
recommend postoperative $\mathrm{PET} / \mathrm{CT}$ imaging in all patients, after complete wound healing.

Clonal T-cells usually occur in the peri-implant effusion. Therefore, BIA-ALCL diagnosis is based on cytology with immunostaining [8]. Direct smears are likely to be insufficient for definitive diagnosis; consequently, it is necessary to aspirate about 20 to $50 \mathrm{ml}$ of effusion and send it fresh to a laboratory. In our series, in 2 cases, in which effusion was collected preoperatively, this approach enabled BIA-ALCL to be diagnosed. In one case, fluid was sent for flow cytometry, which confirmed the initial diagnosis. As T-cell clonality evaluation clarifies T-cell origin of BIA-ALCL, additional molecular tests are particularly required. In uncertain morphological and immunophenotypic features, e.g. in a lymphocyte-rich seroma with few $(<10 \%)$ atypical CD30+ cells, flow cytometryor polymerase chain reaction (PCR)-based methods (e.g., gene scan analysis) allowed evaluation of clonal rearrangement [35]. Besides the benefits of molecular facilities, we share the opinion that for timely diagnosis and costs, they should be limited to the cases of equivocal cytology and immunostaining [36].

All the patients in our study underwent capsulectomy with implant removal, which is a standard treatment of BIA-ALCL [9, 22, 25, 34-39]. In most cases, the procedures were bilateral, which was dictated by esthetic reasons to maintain the symmetry or by patients' and surgeons' concerns. Still, the risk of asymptomatic BIA-ALCL in the contralateral breast is low; according to Clemens et al., it is below 5\% [38]. Little is known about breast reconstruction in BIA-ALCL patients.
We believe immediate breast reconstruction may be justified only in early-stage patients, who are highly determined to undergo such an approach. If a patient expects delayed breast reconstruction, exclusion of lymphoma recurrence is mandatory. Regardless of the timing of breast restoration, autologous tissue or smooth implants should be used rather than textured devices [39].

The differential diagnosis of BIA-ALCL includes systemic ALK-negative ALCL with secondary involvement of the breast. History of breast implantation is crucial. In our study, 1 patient with systemic BIA-ALCL received primary intensive chemotherapy followed by high-dose chemotherapy supported by autologous stem cell transplantation. Afterwards, she underwent surgical bilateral capsulectomies with explantations. This case emphasizes that a patient with breast implants and ALK-negative ALCL diagnosis should have a thorough breast examination, including ultrasound or MRI. Such an approach allows diagnosis of an advanced stage of BIA-ALCL, which, in addition to chemotherapy, requires surgical treatment.

There are several algorithms of BIA-ALCL treatment, which depict an optimal way from diagnosis, via the treatment to the follow-up [9, 22, 34-38, $40,41]$. They are systematically updated and may differ depending on the country-specific healthcare systems. As a consensus of the Polish task force for BIA-ALCL, we proposed 10-point guidelines for the surgeons who use breast implants in esthetics and reconstructive indications (Table III).

The main disadvantage of our study is that we did not assess the risk of BIA-ALCL in Polish population. Lack of reliable data of the number of wom-

Table III. Practical guidelines for breast surgeons for BIA-ALCL diagnosis and management

1. Remember that any post-implantation patient with late-onset, non-inflammatory breast swelling requires BIA-ALCL diagnostic tests

2. Begin the workup with breast and axillary ultrasound, and if in any doubt, send for ancillary breast MRI. Scant or minimal amount (less than $10 \mathrm{ml}$ ) of fluid in the peri-implant capsule is typical

3. In increased peri-implant effusion, perform an ultrasound-guided FNA biopsy. To avoid the implant puncture, use a blunt cannula. Try to collect at least $20 \mathrm{ml}$ of fluid and send fresh for cytology without delay. Take a swab test to exclude infection

4. Core biopsy is mandatory if the breast mass is detected

5. Pinpoint your clinical suspicion of BIA-ALCL in the pathology request and contact the laboratory. Your cooperation with a pathologist will be essential to establish a proper diagnosis

6. Advanced BIA-ALCL patients require a multidisciplinary team approach. Promptly refer each patient with lymph node enlargement or systemic symptoms, such as fatigue, weight loss, fever and night sweats to the tertiary cancer center

7. In patients with local disease (peri-implant effusion and mass limited to the fibrous capsule; no pathological nodes; no systemic symptoms) perform en-bloc total capsulectomy with implant removal. Before the surgery, discuss with the patient the possibility of implant replacement with a smooth or minimally textured surface, and consider a similar approach in the contralateral breast

8. Submit the capsules and peri-implant fluid for definitive pathological assessment. Remember to attach full clinical data in the request (see point 5)

9. Regardless of the BIA-ALCL staging, an oncologist's or hematologist's advice is mandatory for all patients after the surgery. It is to consider adjuvant treatment and set the follow-up schedule

10. Please report the case to the national BIA-ALCL database or task force. In Poland: bia.alcl@ptcho.org

$B I A-A L C L$ - breast implant-associated anaplastic large cell lymphoma, MRI - magnetic resonance imaging, FNA - fine-needle aspiration. 
P. Pluta, A. Giza, M. Kolenda, W. Fendler, M. Braun, C. Chudobiński, J. Chałubińska-Fendler, M. Araszkiewicz, K. Loga, L. Lembas, M. Witkowska, A. Pluta, J. Kolasiński, P. Basta, A. Kołacińska-Voytkuv, S. Cieśla, D. Jesionek-Kupnicka, J. Jankau, M. Kuczyński, E. Kalinka, M. Zadrożny, D. Murawa

en with breast implants in Poland was the reason. The national commercial law ensures confidentiality of the sales data, including the expanders and implants market. Furthermore, our sighting study showed insufficiency of the questionnaire survey on plastic surgery and surgical oncology centers. For these reasons, the incidence of BIA$\mathrm{ALCL}$ in the Polish female population exposed to the breast implant remains unknown. Instead, we estimated the annular incidence of BIA-ALCL in the total Polish female population, excluding women under 30, whom breast implant surgery seldom concerns. In this selective group, the rate was under 0.021/100 000/year. It fulfilled the criteria of rare cancer, defined as malignancies with incidence below 6/100 000/year according to RARECARE - the project Surveillance of Rare Cancers in Europe $[42,43]$. Rare cancers are a significant public health problem, as in total, their various types represent $10-24 \%$ of all tumors diagnosed in Europe - according to European Society for Clinical Oncology [44]. Although the RARECARE registry has not included BIA-ALCL, the risk of inadequate diagnosis and treatment (both under- and overtreatment) also concerns this entity. Moreover, if we consider BIA-ALCL as an iatrogenic disease, any experience of patient care as an understanding of pathogenesis is valuable to avoid such jeopardy in future. It justifies all efforts to create national BIA-ALCL registries and task forces, and boosts international research collaboration.

In conclusion, both breast augmentation and postmastectomy reconstruction are procedures improving quality of life. Oncological safety is crucial in this context. Our study confirmed that in the Polish population, BIA-ALCL remains scarce and has a generally excellent prognosis. We can foresee that due to the withdrawal of roughly textured implants from the market, and thereby exclusion of likely the most potent etiologic factor, the incidence of BIA-ALCL will decrease with time. However, it does not exempt a breast surgeon from informing patients of the risk of BIA-ALCL and further vigilance during their follow-up. We believe practical guidelines proposed in our paper will be a helpful tool to manage BIA-ALCL patients fast and safely.

\section{Acknowledgments}

We thank Dr Guido Libondi from the Department of Surgical Oncology, Cracow, Poland and Dr Daniel Maliszewski from the Department of General Surgery and Surgical Oncology, Slupsk, Poland for participating in a preliminary sighting study.

\section{Conflict of interest}

The authors declare no conflict of interest.

\section{References}

1. International Society of Aesthetic Plastic Surgery. IAPS international survey on aesthetic/cosmetic procedures performed in 2018, https://www.isaps.org/wp-content/ uploads/2019/12/ISAPS-Global-Survey-Results-2018new.pdf [accessed 28 May 2020].

2. Cemal Y, Albornoz CR, Disa JJ, et al. A paradigm shift in U.S. breast reconstruction: Part 2. The influence of changing mastectomy patterns on reconstructive rate and method. Plast Reconstr Surg 2013; 131: 320e-6e.

3. Panchal H, Matros E. Current trends in postmastectomy breast reconstruction. Plast Reconstr Surg 2017; 140: 7S-13S.

4. Scarpa C, Borso GF, Vindigni V, Bassetto F. Polyurethane foam-covered breast implants: a justified choice? Eur Rev Med Pharmacol Sci 2015; 19: 1600-6.

5. Castel N, Soon-Sutton T, Deptula P, Flaherty A, Parsa FD. Polyurethane-coated breast implants revisited: a 30 year follow-up. Arch Plast Surg 2015; 42: 186-93.

6. Keech JA Jr, Creech BJ. Anaplastic T-cell lymphoma in proximity to a saline-filled breast implant. Plast Reconstr Surg 1997; 100: 554-5.

7. Swerdlow SH, Campo E, Pileri SA, et al. The 2016 revision of the World Health Organization classification of lymphoid neoplasms. Blood 2016; 127: 2375-90.

8. Di Napoli A. Achieving reliable diagnosis in late breast implant seromas: from reactive to anaplastic large cell lymphoma. Plast Reconstr Surg 2019; 143: 15S-22S.

9. Clemens MW, Jacobsen ED, Horwitz SM. 2019 NCCN Consensus Guidelines on the diagnosis and treatment of breast implant-associated anaplastic large cell lymphoma (BIA-ALCL). Aesthet Surg J 2019; 39: S3-13.

10. Brown RA, Fernandez-Pol S, Kim J. Primary cutaneous anaplastic large cell lymphoma. J Cutan Pathol 2017; 44: 570-7.

11. Vose J, Armitage J, Weisenburger D; International T-Cell Lymphoma Project. International peripheral T-cell and natural killer/T-cell lymphoma study: pathology findings and clinical outcomes. J Clin Oncol 2008; 26: 4124-30.

12. American Society of Plastic Surgeons. BIA-ALCL Physicians Resources, https://www.plasticsurgery.org/formedical-professionals/health-policy/bia-alcl-physicianresources [accessed 28 May 2020].

13. Thomas A, Link BK, Altekruse S, Romitti PA, Schroeder MC. Primary breast lymphoma in the United States: 1975-2013. J Natl Cancer Inst 2017; 109: djw294.

14. Orphanet. https://www.orpha.net/consor/cgi-bin/OC_Exp. php?lng=EN\&Expert=98841 [accessed 17 August 2020].

15. de Boer M, van Leeuwen FE, Hauptmann M, et al. Breast implants and the risk of anaplastic large-cell lymphoma in the breast. JAMA Oncol 2018; 4: 335-41.

16. Clemens MW, Miranda RN, Butler CE. Breast implant informed consent should include the risk of anaplastic large cell lymphoma. Plast Reconstr Surg 2016; 137: 1117-22.

17. Cardoso MJ, Wyld L, Rubio IT, et al. EUSOMA position regarding breast implant associated anaplastic large cell lymphoma (BIA-ALCL) and the use of textured implants. Breast 2019; 44: 90-3.

18. French National Agency for Medicines and Health Products Safety (ANSM). Décision du 02/04/2019, https://ansm.sante.fr/Decisions/Injonctions-decisions-de-police-sanitaire-sanctions-financieres-interdictions-de-publicite-Decisions-de-police-sanitaire/ Decision-du-02-04-2019-portant-interdiction-de-misesur-le-marche-de-distribution-de-publicite-et-d-utilisation-d-implants-mammaires-a-enveloppe-macro-textu- 
ree-et-d-implants-mammaires-polyurethane-ainsi-queretrait-de-ces-produits [accessed 28 May 2020].

19. Cheson BD, Fisher RI, Barrington SF, et al. Recommendations for initial evaluation, staging, and response as sessment of Hodgkin and non-Hodgkin lymphoma: the Lugano classification. J Clin Oncol 2014; 32: 3059-68.

20. Statistics Poland. Databases, https://stat.gov.pl/ [ac cessed 1 May 2020]

21. Poźniak-Balicka R, Murawa D. Breast implant-associated anaplastic large cell lymphoma (BIA-ALCL). Oncol Clin Pract 2020; 16: 36-9.

22. Marra A, Viale G, Pileri SA, et al. Breast implant-associated anaplastic large cell lymphoma: a comprehensive review. Cancer Treat Rev 2020; 84: 1019-63.

23. Jones P, Mempin $\mathrm{M}, \mathrm{Hu} \mathrm{H}$, et al. The functional influence of breast implant outer shell morphology on bacterial attachment and growth. Plast Reconstr Surg 2018; 142: 837-49.

24. Blombery P, Thompson ER, Jones K, et al. Whole exome sequencing reveals activating JAK1 and STAT3 mutations in breast implant-associated anaplastic large cell lymphoma anaplastic large cell lymphoma. Haematologica 2016; 101: e387-90.

25. Loch-Wilkinson A, Beath KJ, Knight RJW, et al. Breast implant-associated anaplastic large cell lymphoma in Australia and New Zealand: high-surface-area textured Implants are associated with increased risk. Plast Reconstr Surg 2017; 140: 645-54.

26. Ghosh T, Duncavage E, Mehta-Shah N, McGuire PA, Te nenbaum M, Myckatyn TM. A cautionary tale and update on breast implant-associated anaplastic large cell lymphoma (BIA-ALCL). Aesthet Surg J 2020; https://doi. org/10.1093/asj/sjz377.

27. Adams WP Jr, Culbertson EJ, Deva AK, et al. Macrotextured breast implants with defined steps to minimize bacterial contamination around the device: experience in 42,000 implants. Plast Reconstr Surg 2017; 140: 427-31.

28. Adrada BE, Miranda RN, Rauch GM, et al. Breast implant-associated anaplastic large cell lymphoma: sensitivity, specificity, and findings of imaging studies in 44 patients. Breast Cancer Res Treat 2014; 147: 1-14

29. Dashevsky BZ, Gallagher KM, Grabenstetter A, et al. Breast implant-associated anaplastic large cell lymphoma: Clinical and imaging findings at a large US cancer center. Breast J 2019; 25: 69-74.

30. Sá Dos Reis C, Gremion I, Richli Meystre N. Consensus about image quality assessment criteria of breast im plants mammography using Delphi method with radiographers and radiologists. Insights Imaging 2020; 11: 56.

31. Koczkodaj P, Sulkowska U, Gotlib J, Mańczuk M. Breast cancer mortality trends in Europe among women in perimenopausal and postmenopausal age (45+). Arch Med Sci 2019; 16: 146-56.

32. El-Galaly TC, Villa D, Gormsen LC, Baech J, Lo A, Cheah CY. FDG-PET/CT in the management of lymphomas: current status and future directions. J Intern Med 2018; 284: 358-76

33. National Comprehensive Cancer Network (NCCN) clinical practice guidelines in oncology. T-cell lymphomas. Version 1-2020, https://www.nccn.org/store/login/login. aspx?ReturnURL=https://www.nccn.org/professionals/ physician gls/pdf/t-cell.pdf [accessed 28 May 2020].

34. Leberfinger AN, Behar BJ, Williams NC, et al. Breast implant-associated anaplastic large cell lymphoma: a systematic review. JAMA Surg 2017; 152: 1161-8.

35. Mehta-Shah N, Clemens MW, Horwitz SM. How I treat breast implant-associated anaplastic large cell lymphoma. Blood 2018; 132: 1889-98.
36. Gunawardana RT, Dessauvagie BF, Taylor DB. Breast implant-associated anaplastic large cell lymphoma, an under-recognised entity. J Med Imaging Radiat Oncol 2019; 63: 630-8.

37. Jones JL, Hanby AM, Wells $C$, et al. Breast implant-associated anaplastic large cell lymphoma (BIA-ALCL): an overview of presentation and pathogenesis and guidelines for pathological diagnosis and management. Histopathology 2019; 75: 787-96.

38. Clemens MW, Medeiros LJ, Butler CE, et al. Complete surgical excision is essential for the management of patients with breast implant-associated anaplastic large-cell lymphoma. J Clin Oncol 2016; 34: 160-8.

39. Lamaris GA, Butler CE, Deva AK, et al. Breast reconstruction following breast implant-associated anaplastic large cell lymphoma. Plast Reconstr Surg 2019; 143: 51S-8S.

40. Johnson L, O’Donoghue JM, McLean N, et al. Breast implant associated anaplastic large cell lymphoma: The UK experience. Recommendations on its management and implications for informed consent. Eur J Surg Oncol 2017; 43: 1393-401.

41. Locke MB, Lofts J. Variable presentation of anaplastic large-cell lymphoma in patients with breast implants. ANZ J Surg 2017; 87: 789-94.

42. Rare Cancers Europe. About rare cancers. https://www. rarecancerseurope.org/ [accessed 28 May 2020].

43. Casali PG, Trama A. Rationale of the rare cancer list: a consensus paper from the Joint Action on Rare Cancers (JARC) of the European Union (EU). ESMO Open 2020; 5: e000666.

44. Gatta G, Capocaccia R, Botta L, et al. Burden and centralised treatment in Europe of rare tumours: results of RARECAREnet-a population-based study. Lancet Oncol 2017; 18: 1022-39. 